Gefässchirurgie 2014 · 19:13-14

DOI 10.1007/s00772-013-1283-9

Online publiziert: 10. Februar 2014

(c) Springer-Verlag Berlin Heidelberg 2014
B. Lutz ${ }^{1} \cdot$ C. Wieker ${ }^{2} \cdot$ T. Yilmaz $^{3}$

${ }^{1}$ Klinik für vaskuläre und endovaskuläre Chirurgie, Klinikum rechts der Isar der TU München

2 Klinik für Gefäßchirurgie, Universitätsklinikum Heidelberg

${ }^{3}$ Sektion Gefäß- und Endovascularchirurgie, Hospital zum heiligen Geist, Frankfurt

\title{
Wie sieht der Gefäßchirurg von morgen aus?
}

Sehr geehrte Kolleginnen und Kollegen,

als Mitglieder des Jungen Forums der Deutschen Gesellschaft für Gefäßchirurgie und Gefäßmedizin e.V. (DGG) freuen wir uns sehr, das Editorial zur Weiterbildungsausgabe der Gefäßchirurgie schreiben zu dürfen.

Das Junge Forum der DGG wurde im Jahr 2009 im Rahmen des Heidelberger Heißen Eisens beim "HEAT“ (Heidelberger Assistenten Treffen) gegründet und stellt als Interessenvertretung der Assistenzärztinnen und -ärzte in Weiterbildung das Bindeglied zwischen den Gremien und dem Vorstand der DGG, jungen Fachärzt(inn)en und den Assistent(inn) en dar. Ziel soll es hierbei sein, nicht nur die Interessen gegenüber dem Vorstand $\mathrm{zu}$ vertreten, sondern auch aktiv die $\mathrm{Zu}$ kunft der gefäßchirurgischen Gesellschaft mitzugestalten.

Die Schwerpunkte des Jungen Forums der DGG wurden hierfür in vier Bereiche gegliedert, aus welchen sich die vier aktuellen Arbeitsgruppen bildeten. Diese sind die AG Familie, AG Kommunikation, AG Forschung und AG Weiterbildung.

Wie sieht nun der aktuelle gefäßchirurgische Alltag aus, wo bewegt sich die Gefäßchirurgie in Zukunft hin und welche Konsequenz hat dies auf die Weiterbildung in unserem Fach?

Dies sind die essenziellen Fragen, mit denen sich Gefäßchirurg(inn)en in der Weiterbildung täglich auseinandersetzen müssen. Denn der Nachwuchsmangel ist in chirurgischen Fächern weiterhin nicht nur absehbar, sondern auch in den meisten Kliniken bereits allgegenwärtig.

\begin{tabular}{l}
\hline Der Nachwuchsmangel \\
ist in den meisten Kliniken \\
bereits allgegenwärtig \\
\hline
\end{tabular}

„Bis 2020 geht ca. die Hälfte der niedergelassenen Chirurgen und mehr als ein Drittel der Krankenhauschirurgen in den Ruhestand. Sollten alle vakanten Stellen wieder besetzt werden, müssten jährlich ca. 10-12 Prozent der Medizinstudierenden eine Karriere in der Chirurgie starten. Nach aktuellen Schätzungen wollen maximal nur 5 Prozent der Absolventen die chirurgische Laufbahn einschlagen." [1]

Die Chirurgie muss folglich in Zukunft gute Anreize für Neueinsteiger bieten. Große Kliniken mit breiten Angeboten oder Spezialisierungen sind meist attraktiver für Bewerber, als kleinere Kliniken. Durch das logistisch und politisch eingeschränkte Operationsangebot ist der Ausbildungsstand der Weiterbildungsassistent(inn)en dort begrenzt, was den Mangel an qualifizierten Mitarbeitern in $\mathrm{Zu}$ kunft noch verstärken wird. Die Qualität der Ausbildung sollte aber nicht standortabhängig sein. Wichtig ist es daher auch für kleinere Kliniken, auf dem neusten Stand zu bleiben.

Minimal-invasive Verfahren sind seit vielen Jahren in der gesamten Medizin auf dem Vormarsch. Sie ermöglichen indikationsgerechte Therapien auch bei multimorbiden oder sehr alten Patienten, so- dass therapeutische Grenzen erst sehr spät erreicht werden. Im Bereich der Gefäßchirurgie haben sich somit endovaskuläre Verfahren weitläufig etabliert.

Welche Konsequenz hat dies für den sich in der Weiterbildung befindenden Gefäßchirurgen von morgen?

Durch die Zunahme der endovaskulären Verfahren, treten einige konventionelle Therapien in den Hintergrund, beispielsweise die offene Therapie von Aortenaneurysmen. Schon heute zeichnet sich jedoch ab, dass die Komplikationen der endovaskulären Verfahren durchaus eine klassische, chirurgische Herangehensweise erfordern. Dies beweist, dass die endovaskuläre Therapie in die Hand von Gefäßchirurgen gehört, die im Zweifelsfall die entstehenden Komplikationen selbstständig beherrschen können. Angehende Gefäßchirurg(inn)en werden nunmehr vor der Schwierigkeit stehen, den Komplikationen von endovaskulären Verfahren nicht gewachsen zu sein. Konventionelle, etablierte Verfahren dürfen daher nicht in Vergessenheit geraten, um die Patientenversorgung sowohl in Zentren der Maximalversorgung als auch in der Peripherie weiterhin sicherstellen zu können. Es stellt sich die Frage, ob die ganzheitliche Ausbildung zum Gefäßchirurgen, der den Spagat zwischen neuartigen Prozeduren und etablierten Methoden, die nicht ins Hintertreffen geraten sollen, möglich ist. Bei einem sich so rasant fortentwickelnden Fach wie der Gefäßchirurgie müssen sich somit auch die Weiterbildungsinhalte anpassen. 
Aufgrund des Nachwuchsmangels und zunehmenden finanziellen Einsparungen in den Kliniken ist es schwierig geworden, eine qualitativ gute Weiterbildung anzubieten. Der DRG-orientierte Patientendurchlauf und der Dokumentationsaufwand sind gestiegen, die Patienten werden älter, die Arbeitsschritte aufwendiger und folglich auch die Arbeitsbelastung ohne Veränderung des Stellenschlüssels. Man versucht als Weiterbildungsassistent das Rad des klinischen Alltags am Laufen zu halten, zumeist mit Überstunden, wodurch wenig Zeit für die theoretische und praktische Ausbildung oder die Forschung bleibt. Diese Aufgaben fallen dann in der Freizeit an, was das chirurgische Arbeiten für den potenziellen Nachwuchs unattraktiv macht. Die Realität des chirurgischen Alltags schreckt Studierende $\mathrm{ab}$.

Um eine Veränderung in eine positive Richtung zu bewirken, ist Umdenken und schnelles Handeln unumgänglich. Das Bewusstsein über die neuen Herausforderungen für die Weiterbildungsassistent(inn)en hat dazu geführt, dass eine neue Weiterbildungsordnung entstand, die eine wichtige Weichenstellung für die Zukunft unseres Faches darstellt. Neben den Inhalten dieser neuen WBO möchte das Junge Forum der DGG folgende Ideen mit einfließen lassen:

- Einführung eines Mentoring-Programms ab dem 1. Tag der Weiterbildung mit regelmäßigem Feedback.

- Regelmäßige Kontrollen des Weiterbildungsstandes durch Weiterbildungsbeauftragte und individuelle Verbesserungsvorschläge in Zielvereinbarungsgesprächen sowie deren Umsetzung.

- Strukturierte Assistentenrotationen zwischen kleinen und großen Kliniken, um die Inhalte des Weiterbildungscurriculums zu erfüllen.

Die Gefäßchirurgie als einer der jüngsten, selbstständigen chirurgischen Fachbereiche bietet heute einen Reichtum an Therapieverfahren an. Daher steht der Weiterbildungsbefugte vor der Aufgabe, diese Inhalte fair und strukturiert den Assistenten in der Weiterbildung zu vermitteln. Neben der Weiterbildung und Freiräumen für Forschung sollten auch pri- vate Themen wie Work-Life-Balance und Familienplanung berücksichtigt werden. Das Junge Forum der DGG (http://www. junges-forum-dgg.de) vertritt diese Interessen der Assistenten in der Gefäßchirurgie und hofft weiterhin ein erfolgreiches Bindeglied zwischen Assistenten und Weiterbildungsbeauftragten darzustellen.

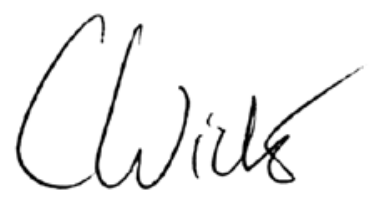

Carola Wieker

(Sprecherin Junges Forum),

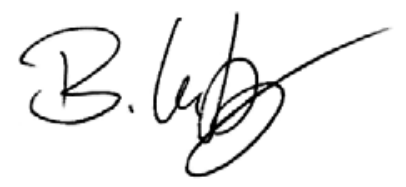

Brigitta Lutz

(stellvertretende Sprecherin) und

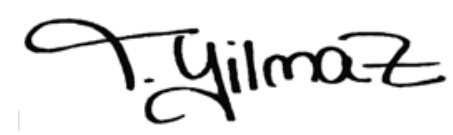

Tunay Yilmaz

(AG-Leiterin Weiterbildung)

\section{Korrespondenzadressen}

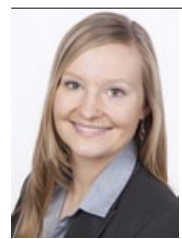

B. Lutz

Klinik für vaskuläre und endovaskuläre Chirurgie, Klinikum rechts der Isar der TU München

Ismaninger Str. 22, 81675 München

b.lutz@lrz.tum.de

C. Wieker
Klinik für Gefäßchirurgie,
Universitätsklinikum
Heidelberg
Im Neuenheimer Feld 110,
69120 Heidelberg

Interessenkonflikt. Die korrespondierende Autorin gibt für sich und ihre Koautorinnen an, dass kein Interessenkonflikt besteht.

\section{Literatur}

1. Krüger M (2008) Die chirurgische Weiterbildung in Deutschland und die Rolle des Weiterbilders. Eine Assistentensichtweise. Chirurg BDC 47:301-304 